\title{
A 110 MG cyclotron harmonic in the optical spectrum of RX J1554.2+2721
}

\author{
A. D. Schwope ${ }^{1}$, M. R. Schreiber ${ }^{1}$, and P. Szkody ${ }^{2}$ \\ 1 Astrophysikalisches Institut Potsdam, An der Sternwarte 16, 14482 Potsdam, Germany \\ e-mail: aschwope@aip.de \\ 2 Dept. of Astronomy, Box 351580, University of Washington, Seattle, WA 98195, USA
}

Received 22 December 2005 / Accepted 28 February 2006

ABSTRACT

\begin{abstract}
We report the detection of a $110 \mathrm{MG}$ cyclotron harmonic in the SDSS-spectrum of the magnetic cataclysmic variable (MCV) RXJ1554.2+2721. This feature was noted earlier by others but remained unexplained. The wide spectral coverage of the new spectrum together with the earlier detection of a Zeeman split Ly $\alpha$ line in a field of 144 MG makes the identification almost unambiguous. We propose to explain the non-conforming UV-optical photospheric temperature of the white dwarf by an as yet unobserved cyclotron component in the ultraviolet which also could significantly contribute to the overall energy balance of the accretion process.
\end{abstract}

Key words. stars: novae, cataclysmic variables - stars: individual: RX J1554.2+2721- radiation mechanisms: thermal accretion, accretion disks - while dwarfs - binaries: close

\section{Introduction}

AM Herculis stars, also termed polars, form the sub-group of cataclysmic variable stars hosting a strongly magnetic white dwarf primary as accretor. The field prevents the formation of an accretion disk and keeps both stars in synchronous rotation. Accretion happens via Roche-lobe overflow and streams/curtains down to the polar regions of the white dwarf. The accretion plasma, typical temperatures are $k T \simeq$ $10 \mathrm{keV}$, cools via thermal plasma radiation in the X-ray domain and via cyclotron radiation. Depending on the field strength in the accretion region, which is between 10 and $60 \mathrm{MG}$ for most of the polars, the cyclotron spectrum emerges at IR or optical wavelength. Cyclotron radiation in the UV is equally possible but observed only in a few cases due to the sparcity of highfield systems (see Rosen et al. 2001; Gänsicke et al. 2001, and Nogami et al. 2002 for results on QS Tel, AR UMa, and UZ For, respectively).

RX J1554.2+2721 was identified as a CV in the Hamburg Quasar Survey (Jiang et al. 2000) and independently by Tovmassian et al. (2001, henceforth TEA01) as the optical counterpart of a soft RASS source. Its magnetic nature was suggested by Tovmassian et al. who also tentatively identified low frequency flux variations as cyclotron harmonics in a field of $\sim 30 \mathrm{MG}$. They also recognized its period in the CV period gap. Thorstensen \& Fenton (2002, henceforth TF02) determined an accurate orbital period of $P_{\text {orb }}=151.865 \mathrm{~min}$ right in the centre of the period gap and estimated the distance to the system on the basis of spectral features of the secondary star to be roughly $210 \mathrm{pc}$. They also noted a pronounced double-humped structure of the I-band light curve due to ellipsoidal modulation of the secondary and a spectral hump of possible magnetic origin at around $5000 \AA$. Finally, Gänsicke et al. (2004, henceforth GEA04) reported the detection of Zeeman split Ly $\alpha$ absorption lines in a HST-STIS snapshot spectrum. The UV data

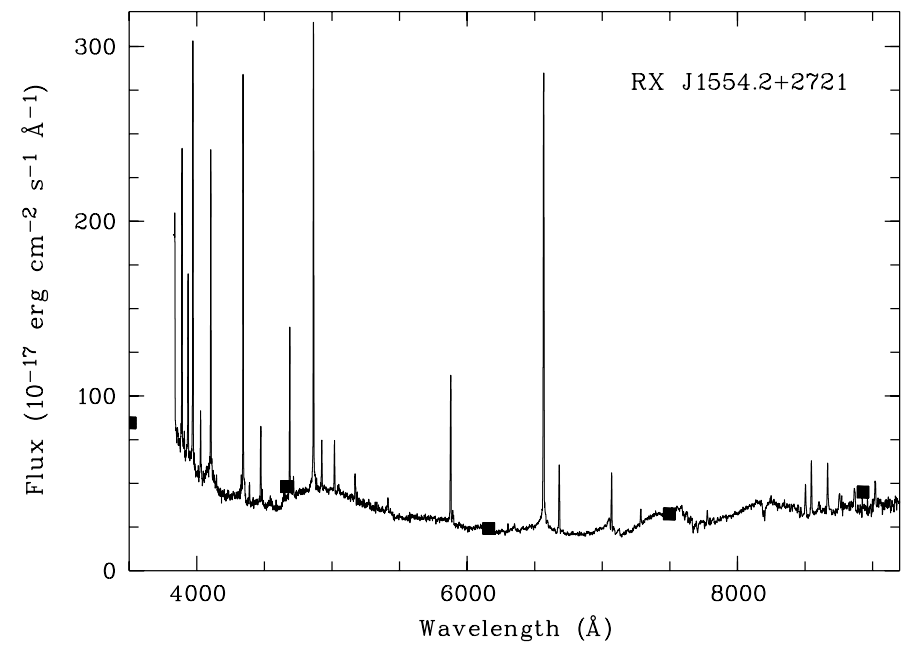

Fig. 1. SDSS-spectrum of RXJ1554.2+2721 obtained May 8, 2005. Also shown is the ugriz photometry obtained June 22, 2003.

were successfully modeled with a centered dipole model with polar field strength $144 \mathrm{MG}$ and photospheric temperature of $17500 \mathrm{~K}$, thus making RXJ1554.2+2721 only the third polar with a field strength in excess of $100 \mathrm{MG}$. The combined optical/UV spectral energy distribution, however, suggested a significantly higher temperature of about $23000 \mathrm{~K}$.

\section{A new SDSS spectrum of RX $\mathbf{J 1 5 5 4 . 2 + 2 7 2 1}$}

Our review on RX J1554.2+2721 is motivated by a single spectrum taken by the SDSS on May 8, 2005. The spectrum of the object with designation SDSS J155412.33+272152.4, obtained with an exposure time of $46 \mathrm{~min}$, is reproduced in Fig. 1. Also shown in the figure is the SDSS photometry 
obtained June 22, 2003, with $u=17.60, g=17 \mathrm{~m} .55, r=$ $17.70, i=16.95$, and $z=16.19$, which shows very little deviation from the spectroscopy. For details of the SDSS project the reader is refered to Adelman-McCarthy et al. (2006), Fukugita et al. (1996), Gunn et al. (2006), and York et al. (2000).

The accumulated phase uncertainty at the time of the spectral observations, i.e. after 13456 cycles according to the ephemeris of TF02 is $\Delta \phi=0.75$, hence we cannot assign a proper orbital phase to the spectrum. The main features of the spectrum are unchanged with respect to the observations by TF02 and TEA01, the prevalence of the M4 secondary in the red spectral range, a hump centered on $\sim 5000 \AA$, prominent high-state emission lines of $\mathrm{H}$ and $\mathrm{He}$ and a spectral upturn at the blue end.

The new SDSS spectrum has a continuum flux level very similar to the March 2001 low-state spectrum by TF02 (their Fig. $3 \mathrm{~b}$ ) but a very different emission line spectrum containing even lines of ionized helium. The low-state spectrum of TF02 has $\sim 20 \%$ less flux in the range $5000-7000 \AA, 10 \%$ more flux at $4600 \AA$ and matches exactly at $7600 \AA$, where mainly the secondary contributes. The pronounced difference in the line spectrum between TF02 low state and SDSS suggests the presence of a soft X-ray ionizing source at the time, when the SDSS spectrum was taken, hence a certain level of accretion. The SDSSspectrum was thus not obtained in a low accretion state although the continuum flux level might be regarded as indicative of a low state.

The May 2001 high-state spectrum of TF02 has a very similar line spectrum compared to the SDSS spectrum but an enhanced continuum flux by about $20-30 \%$. The red part of the high-state spectrum by TEA01 (their Fig. 4b) matches exactly the new SDSS spectrum while the blue part of their spectrum taken one night later is again about $25 \%$ brighter. Taken together, neither the orbital variability nor the rather frequent changes between high and low states seem to have a pronounced influence on the system's brightness (although one must admit that highstate photometry in the blue part of the spectrum is missing).

The atomic emission lines in the SDSS-spectrum seem to be unusually sharp and peaked. They can be fit with the superposition of a broad and a narrow Gaussian line with $F W H M$ $2000 \mathrm{~km} \mathrm{~s}^{-1}$ and $250-300 \mathrm{~km} \mathrm{~s}^{-1}$ (after deconvolution with the instrumental profile). These narrow lines appear wider than the narrow emission lines in other polars which are of reprocessed origin from the secondary $\left(F W H M \sim 100 \mathrm{~km} \mathrm{~s}^{-1}\right)$ but velocity smearing is likely be responsible for the relative large width. The broad line clearly indicates an origin in an accretion stream.

The very feature which makes the spectrum of RXJ1554.2+2721 outstanding is the hump centered on $\sim 4950 \AA$, which we regard as a cyclotron emission line, either the cyclotron fundamental or a low cyclotron harmonic. This feature was present in the spectra of TEA01 and TF02 too but didn't show up so prominently due to the shorter wavelength coverage and blending with atomic emission lines.

\subsection{The cyclotron harmonic at $4950 \AA$}

In oder to isolate the cyclotron component we subtracted a scaled template M-dwarf spectrum and the $20000 \mathrm{~K}$ magnetic white dwarf model spectrum by GEA04 adjusted to the flux level around $4600 \AA$. For subtraction of the template M-dwarf we initially used the same spectrum of G1447 (M4+) as TF02. The SDSS spectrum required a scaling parameter $20 \%$ different from TF02 which led to a slightly larger distance estimate, $235 \mathrm{pc}$ instead of $210 \mathrm{pc}$. We finally used a spectrum of GJ 268

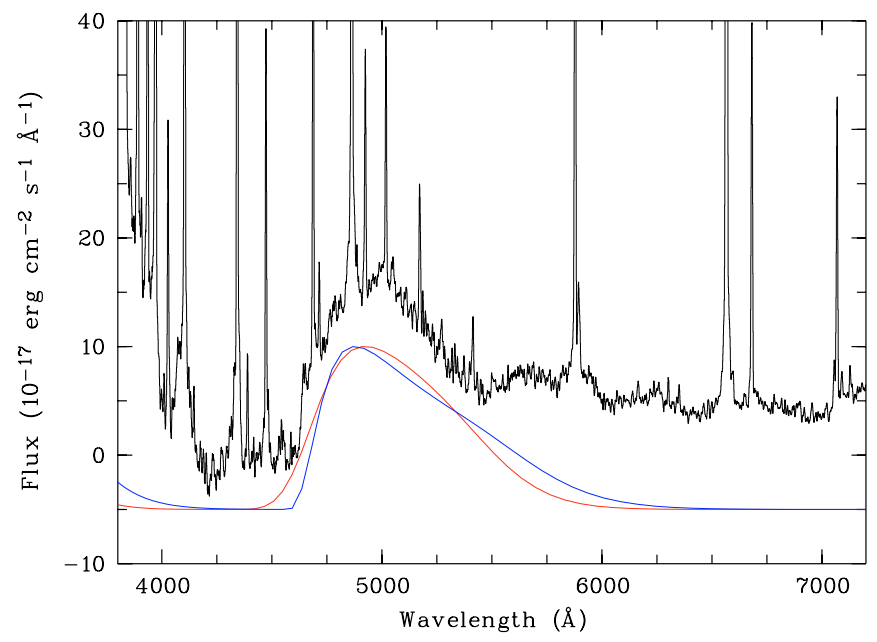

Fig. 2. Cyclotron spectrum of RX J1554.2+2721 computed by sutraction of suitably scaled M-dwarf and white dwarf spectra. The model spectra for 5 and $10 \mathrm{keV}$ are plotted with an offset of -5 units.

(M4.5) available to us comprising a wavelength range similar to that of the SDSS-spectrum. The difference spectrum is regarded to mainly consist of cyclotron radiation but might be affected by Zeeman absorption and might have additional contributions from the recombination continuum. On the basis of just one new spectrum the latter two components cannot be quantified.

The difference spectrum is reproduced in Fig. 2. It shows a single cyclotron harmonic rising sharply at $4600 \AA$ and dropping of at $\sim 5450 \AA$. The red continuum has more flux than the blue continuum between $4150-4600 \AA$. The use of a cooler $15000 \mathrm{~K}$ white dwarf instead of the $20000 \mathrm{~K}$ does not cure the problem. Either there is an emission component in the red part of the spectrum or the blue part is affected by Zeeman absorption. Again, on the basis of just one spectrum we cannot decide between the alternatives but our main conclusions are not affected by these uncertainties.

The lack of any neighboring cyclotron harmonic excludes any low-field/high-harmonic interpretation of the observed structure. The $144 \mathrm{MG}$ field determined by GEA04 suggests an identification with either the cyclotron fundamental or the second harmonic. If it would be the fundamental then the derived field strength would be $220 \mathrm{MG}$. This exceeds the implied polar field strength by a large amount and is regarded as the less likely, although not impossible interpretation. If for instance the photospheric spectrum would be obtained under an unfavourable viewing geometry or the field structure deviate from the centered dipole model, as many polars do, the assumed polar field strength can be very much different from the real value. However, unless other evidence is available the identification with the second harmonic seems plausible.

We have modeled the harmonic with isothermal plasma cyclotron radiation using the code described in previous papers (Schwope et al. 1990). Two model curves with slightly different field strength $B$, plasma temperature $k T$, aspect angle $\theta$, but the same density parameter $\Lambda$ are also shown in Fig. 2. The density parameter $\Lambda=l \omega_{\mathrm{p}}^{2} / c \omega_{\mathrm{c}}=$ $\left.5 \times 10^{4}\left(l / 10^{5} \mathrm{~cm}\right)\left(n_{\mathrm{e}} / 10^{1} 6 \mathrm{~cm}^{-} 3\right)(110 \mathrm{MG}) / B\right)$ essentially determines where the spectrum turns from the low-frequency optically thick to the high-frequency optically thin part. The angle $\theta$ is the angle between the line of sight and the magnetic field. The narrower line was calculated for $k T=5 \mathrm{keV}, B=108 \mathrm{MG}$, $\theta=60^{\circ}, \log \Lambda=1$, the broader line for $10 \mathrm{keV}, 113 \mathrm{MG}, 70^{\circ}$, 
and $\log \Lambda=1$. The width of individual cyclotron lines is a function of $\theta$ and $T$, but a scatter in these values and, in particular, a scatter in $B$ may further broaden individual lines. We cannot discern between these different broadening mechanisms and the models were calculated with the most simple assumptions, a homogeneous plasma with $\theta$ adjusted to match the observed width of the line.

\section{Discussion}

\subsection{The UV-optical spectral energy distribution}

There is evidence that the one single cyclotron harmonic is not fully representative of the cyclotron spectrum of RX J1554.2+2721 in the main accretion region. The value of the density parameter used for the fits shown in Fig. 2 was extremely $\operatorname{low}, \log \Lambda=1$. This value is comparable to or even lower than those in the recently discovered LARPs (Low Accretion Rate Polars, Schwope et al. 2002, Schmidt et al. 2005) with permanent low accretion rates probably fed from a wind. RX J1554.2+2721 clearly does not belong to this elusive class of objects. It was a RASS source, it shows prominent atomic emission lines of highly ionized species, and high-state Doppler tomograms indicate Roche-lobe overflow (TEA01).

While RX J1554.2+2721 seems to belong to the normal accreting polars displaying high and low accretion states, the absence of a pronounced increase in the continuum when switching from high to low states is puzzling. One distinct possibility is that the main accretion region is continuously hidden from our view due to an unfavourable geometry. In this model the observed cylotron harmonic would belong to a secondary region with much lower accretion rate (as in VV Pup or UZ For, see Wickramasinghe et al. 1989; Schwope \& Beuermann 1997; Schwope et al. 1990). This scenario can be tested by a decent $\mathrm{X}$-ray observation in a high accretion state (indicated by the presence of strong atomic emission lines) which should result in a very low X-ray signal.

Here we discuss shortly an alternative model. Beuermann (2004) discussed a synthesized spectrum of AM Her composed of a multi-temperature, multi-density plasma due to contributions from regions with a wide range of specific mass accretion rates. Following his arguments we may expect the major part of the cyclotron radiation emitted at higher harmonic number in the ultraviolet. Assuming a similar scenario for RX J1554.2+2721 we can give a rough estimate of those suspected contributions from the ultraviolet-to-optical spectral energy distribution shown in Fig. 3. The two observed spectra shown there are the STISspectrum of GEA04 and the SDSS spectrum. GEA04 noticed that a higher temperature is needed to match the SED than derived from just fitting the UV-spectrum. We argue that the inclusion of a cyclotron component with spectral parameters that one usually encounters in high-state polars solves the discrepancy. We illustrate this with a composite spectrum consisting of the scaled M-dwarf GJ 268, the $20000 \mathrm{~K}$ magnetic white dwarf model of GEA04 and two cyclotron models, one with the extreme small density parameter $\log \Lambda=1$, the other with the more typical parameter $\log \Lambda=5$. The synthetic spectrum is not meant to be a proper fit of the data, since there are too many uncertainties in the spectral decomposition ${ }^{1}$ but merely an illustration of the possible spectral composition. A proper composed

1 The main uncertainties are: - the optical and UV spectra were not taken simultaneously; - there is no phase information for either of the two spectra; - we just used two cyclotron models instead of a continuum.

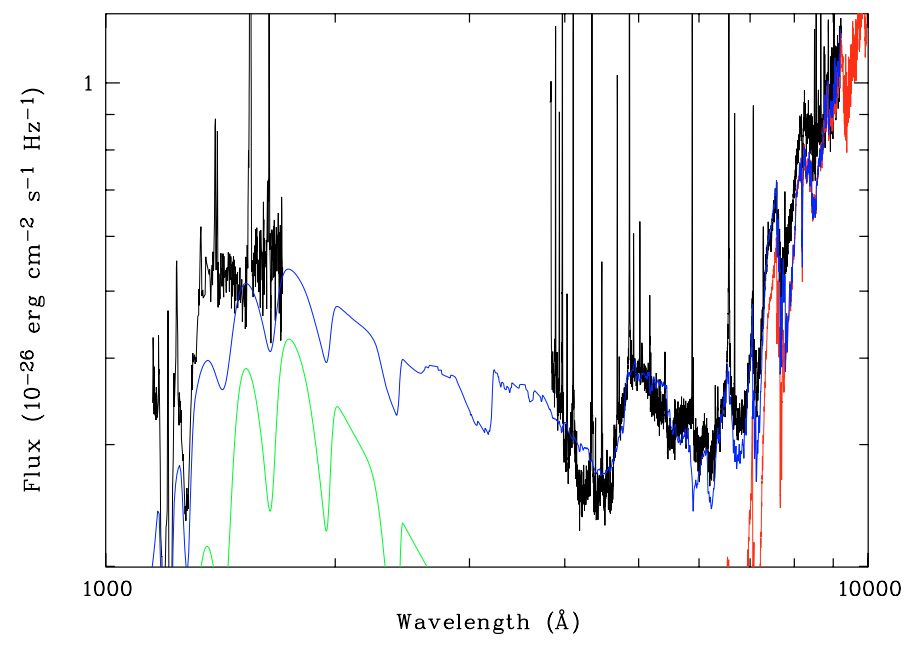

Fig. 3. Combined optical/ultraviolet spectrum of RX J1554.2+2721 using the STIS spectrum of GEA04 and the new SDSS spectrum. The composite model spectrum shown in blue consists of a suitably scaled M-star spectrum (red), two cyclotron model spectra (green) and the $20000 \mathrm{~K}$ white dwarf model of GEA04.

synthetic spectrum will probably explain the upturn at the blue end of the SDSS-spectrum, where the third harmonic starts to rise. The integrated flux of our composite cyclotron spectrum is $F=3 \times 10^{-12} \mathrm{erg} \mathrm{cm}^{-2} \mathrm{~s}^{-1}$, a factor 30 higher than just the flux in the second harmonic and of the same order as the ROSAT $\mathrm{X}$-ray flux in the $0.1-2.4 \mathrm{keV}$ band (TEA01). Neglecting the UV-cyclotron component would result in a heavily biased energy balance of the accretion process for this particular source.

\section{Conclusions}

We have presented an analysis of a new spectrum of RX J1554.2+2721 obtained May 2005 during the SDSS. It shows a pronounced cyclotron hump which could be used to measure the field strength in the accretion plasma and thus gives an explanation to the spectral humps already noticed by TEA01 and TF02.

At a field strength of $110 \mathrm{MG}$ one can access the very low harmonic numbers usually hidden in the infrared or far infrared. This gives access to those parts of a structured accretion region with very low specific mass accretion rates. We propose to explain the non-conforming temperature estimates from UV and optical spectroscopy by a missed cyclotron component in the ultraviolet, which carries most of the cyclotron luminosity. Neglecting such a component could result in a heavily biased energy balance of the accretion process towards thermal plasma radiation in the X-ray regime. A full decomposition of the spectrum requires low-resolution spectroscopy/spectrophotometry with full phase coverage, both in the optical and the ultraviolet.

Acknowledgements. This project is supported by the Deutsches Zentrum für Luft- und Raumfahrt (DLR) under contract no. FKZ 50 OR 0404 (MRS) and by NSF grant AST 97-30792 (PS).

Funding for the Sloan Digital Sky Survey (SDSS) has been provided by the Alfred P. Sloan Foundation, the Participating Institutions, the National Aeronautics and Space Administration, the National Science Foundation, the US Department of Energy, the Japanese Monbukagakusho, and the Max Planck Society. The SDSS Web site is http://www.sdss.org/

The SDSS is managed by the Astrophysical Research Consortium (ARC) for the Participating Institutions. The Participating Institutions are The University of Chicago, Fermilab, the Institute for Advanced Study, the Japan Participation Group, The Johns Hopkins University, the Korean Scientist Group, Los Alamos 
National Laboratory, the Max-Planck-Institute for Astronomy (MPIA), the Max-Planck-Institute for Astrophysics (MPA), New Mexico State University, University of Pittsburgh, University of Portsmouth, Princeton University, the United States Naval Observatory, and the University of Washington.

\section{References}

Adelman-McCarthy, J., et al. 2006, ApJS, in press [arXiv: astro-ph/0507711]

Beuermann, K. 2004, ASPC, 315, 187

Fischer, A., \& Beuermann, K. 2001, A\&A, 373, 211

Fukugita, M., Ichikawa, T., Gunn, J. E., et al. 1996, AJ, 111, 1748

Gänsicke, B., Schmidt, G., Jordan, S., \& Szkody, P. 2001, ApJ, 555, 380
Gänsicke, B., Jordan, S., Beuermann, K., et al. 2004, ApJ, 613, L141 (GEA04) Gunn, J. E., et al. 2006, AJ, submitted

Nogami, D., Gänsicke, B., \& Beuermann, K. 2002, ASPC, 261, 159

Jiang, X. J., Engels, D., Wei, J. Y., Tesch, F., \& Hu, J. Y. 2000, A\&A, 362, 263

Rosen, S. R., Rainger, J. F., Burleigh, M. R., et al. 2001, MNRAS, 322, 631

Schwope, A. D., \& Beuermann, K. 1997, AN, 318, 111

Schwope, A. D., Beuermann, K., \& Thomas, H.-C. 1990, A\&A, 230, 120

Schwope, A. D., Brunner, H., Hambaryan, V., et al. 2002, ASPC, 261, 102

Schmidt, G. D., Szkody, P., Vanlandingham, K. M., et al. 2005, ApJ, 630, 1037

Thorstensen, J., \& Fenton, W. H. 2002, PASP, 114, 74 (TF02)

Tovmassian, G. H., Greiner, J., Zharikov, S. V., Echevarria, J., \& Kniazev, A. 2001, A\&A, 380, 504 (TEA01)

Wickramasinghe, D. T., Ferrario, L., \& Bailey, J. 1989, ApJ, 342, L37

York, D. G., Adelman, J., Anderson, J. E., et al. 2000, AJ, 120, 1579 